\title{
Elaboración de la unidad didáctica: ¿es tu nariz capaz de explicarte qué es un gas?
}

Preparation of the didactic unit: cis capable of your nose is a gas explain?

William Fernando Moreno Torres

Universidad Distrital Francisco Jose de Caldas. wfmorenot@correo.udistrital.edu.co, wfmorenot@estudiante.udistrital.edu.co

\section{Resumen}

En la realización de esta unidad didáctica se pensó en una cosa que tal vez no se había considerado fuera del simple aprendizaje del concepto de los gases; la pretensión principal de la unidad didáctica es resaltar la importancia en que ésta forma de comprensión, de análisis y de síntesis, se puede utilizar para practicarlo, para utilizarlo de forma en que la mayoría de nuestra comunidad sepa de que estamos hablando y se interese por asumir este conocimiento en beneficio general y personal. En pocas palabras y aunque la unidad no se ha aplicado, lo que se pretende es a tener una ciencia accesible a la mayoría de las personas, que sin importar el nivel de escolaridad por lo menos conozca algo nuevo y que le de ideas para aplicarlo en su cotidianidad.

\section{Palabras clave}

Unidad Didáctica, Gases, Conocimiento Científico, Enseñanza, Comunidad.

\section{Abstract}

In carrying out this teaching unit is thought of something that perhaps had not been considered beyond the simple leaming of the concept of the gases, the main daim of the teaching unit is to highlight the importance that this form of understanding, analysis and synthesis can be used to practice, to use it in the majority of our community know that we are talking about and interested in assuming this knowledge for the benefit and general personnel. In a nutshell, and although the unit has not been implemented, which is targeted to have a science accessible to most people, regardless of educational level is kept at least something new in your head to your home.

\section{Keywords}

Unidad Didáctica, Gases, Science, Education, Community.

\section{Introducción}

Esta ponencia se basa en el modelo didáctico de la Resolución de Problemas (RP), el cuál en los últimos tiempos ha dado varias luces sobre el aprendizaje de las ciencias en la formación educativa formal; sin embargo, con todo esto pienso, como otros autores (Mora, García, 2003), que este modelo carece de algunas características que complementańan mejor su propósito en la consolidación de modelo didáctico.

Queremos mencionar que existe una tendencia a definir problema, en la manera en que el estudiante entienda que los conceptos teóricos de las ciencias tienen un propósito en la solución de problemas cotidianos que se presentan en la sociedad, y espećficamente en tres dases que consideramos importantes, la producción, las relaciones sociales con sus matices y, la 
experimentación científica (Mao, 1963); que no se reduce a la mera aplicación de fórmulas o metodología estandarizadas de ejercicios colocado por el profesor o el libro de texto; en esta dirección creo que la discusión no solamente se debe orientar en definir que es un "problema" y un "ejercicio de aplicación", sino que reside en la forma de practicar, es decir en cómo utilizamos estos conceptos teóricos que adquirimos en la formación escolar generalmente como enunciados que solucionan mágicamente los puntos de un quiz o un examen; así que uno de los propósitos de esta unidad es dar elementos e hipotetizar que en un ambiente de practica más ligado a las condiciones concretas del estudiantado, éste puede dar un salto en su conciencia y empeñarse en formarse de tal forma que motive más que una transformación personal sino una transformación en comunidad.

De esta manera, puede que estén reestructuradas ciertas ideas fundamentales de la didáctica, como puede que no, pero lo central es incentivar el debate en cuanto la forma real en que se debe enseñar, teniendo en cuenta las condiciones concretas de nuestro país y sistema educativo.

Para cumplir este propósito quiero ir respondiendo las siguientes preguntas paso a paso, plasmándolo poco a poco en la elaboración de la unidad didáctica para entender el concepto de los gases, daro está que el objetivo es que se pueda tomar de forma general para cualquier concepto dentro del ámbito de las ciencias; así que las preguntas que orientan mi ponencia serían las siguientes: ¿qué determina la conciencia del hombre?, ¿qué es lo fundamental la teoría o la práctica?, ¿qué tipo de practicas existe actualmente en la sociedad? ¿la RP que problemas tiene y auál debería ser su solución posible? Y ¿qué modelo de RP se debería aplicar en nuestra situación concreta?

\section{Desarrollo}

¿Qué determina la conciencia del hombre? Diré esta afirmación, "la vida determina la conciencia del hombre y no la conciencia determina la vida del hombre" (Marx, Engels, 1846), esta frase podría producir estupor, pero no se asusten, es apenas la base de todo lo que ha desarrollado el hombre históricamente; trataré de desglosarla, materialmente el hombre a medida que desarrolla su productividad va creando representaciones mentales que explican su diario vivir, a partir de su actividad, su mente se va moldeando, el artista piensa como un artista, un biólogo como un biólogo, y esto es porque la actividad que realiza es espećfica y determinada por sus condiciones sociales; de manera recíproca el hombre va plasmando su conciencia a medida que desarrolla su actividad productiva, a medida que trabaja; lo que fundamenta la conciencia del hombre es su actividad productiva, actividad productiva que se desenvuelve hoy actualmente con su profesión.

Cuando hablamos actualmente de profesión, hay que tener en auenta de dónde viene tal concepto, es decir, debemos comprender aunque sea, generalmente el origen de esta división que explicita nuestro lenguaje en la forma y modo de producir; para entender esto bastaría comprender el papel primordial que ejerce la división del trabajo dentro de la formación de la conciencia del hombre. Para desarrollar la idea partamos del desarrollo productivo del hombre, es decir de ser recolector, cazador y pescador, se desarrollaron mejores armas y técnicas de sobrevivencia, de este desarrollo se dio un salto hacia la domesticación de animales y posteriormente hacia la agricultura; paralelamente en esto se iban generando una división del hombre, y en realidad estos desarrollos generaron acumulación, centralmente en actividades que involucraban más al hombre que a la mujer, después de ello la acumulación generó alguna forma de propiedad, y para obtener más acumulación se dividió la forma de trabajo y su distribución, en la dirección en que algunos producían y otros administraban, de las diferentes fases en que se desenvolvió esta economía y bajo estas caracteństicas se desprendió entonces el comercio y la industria, que a sus necesidades necesitaban entonces dividir de una manera más eficaz su producción, tanto así que se han generado hombres que trabajan la mente, ó en palabras mas conocidas en nuestro ámbito 
"intelectuales", y estos hombres producen y se desenvuelven en una actividad económica que determina y refleja a su vez su forma de pensar.

En el ámbito estudiantil, lo que hace el estudiante refleja en si su forma de pensar y también el carácter que tiene su oficio, la cultura del facilismo, del menor esfuerzo es reflejo entonces del objetivo y el alcance de ser estudiante en Colombia, entonces cabe esperar que el cambio del estudiante en su oficio depende necesariamente del cambio de las condiciones sociales que puedan ocurrir, y bajo esta lógica sería necesario exigir un cambio de de raíz en las condiciones materiales de la vida de la sociedad colombiana; sin embrago, estos cambios se pueden expresar con el agudizamiento de las contradicciones que engendran las mismas condiciones materiales, y por ende no es sano esperar a que cambie "por arte de magia" todo y luego si empezar a tomar medidas, sino todo lo contrario, desarrollar un cambio en el oficio estudiantil de tal forma que se agudice lo viejo con lo nuevo y estar preparados para el futuro cambio, en esta unidad didáctica, el estudiante no solamente se dedica a "aprender", sino también a interaccionar con su comunidad en un esfuerzo por enseñarle, por explicarle de un modo llamativo y comprensible lo que preparó durante su formación escolar, y junto con esto mirar que su oficio no solamente se quede en "recibir" sino también en "dar", cambiando de por sí su "actividad cotidiana", generando pensamiento crítico (The National Academy Press, 2000).

¿Qué es lo fundamental la teoría o la práctica? Debemos entender que estos dos aspectos hacen parte de una contradicción, la teoría es la que trata de explicarnos y predecir la realidad, y la práctica es el puente que conecta la teoría con la realidad, y por ende deja de ser solo ideas y pasan a ser hechos; es decir, nos enfrentamos entonces una contradicción entre las ideas y la materia en esencia, y como tal debemos empezar a esdarecer aual de ellas es principal y cuál de ellas es secundaria, en que momentos se revisten de esta forma $y$, finalmente cuál es la que determina decisivamente sobre la otra, es decir, ccómo se relacionan? Con la postura sustentada anteriormente, se puede deducir que las bases materiales de la vida determina la conciencia 0 ideas del hombre, por ello la base material del conocimiento, que es a lo que nos referimos en esta pregunta, salta a tener un aspecto importante dentro de la práctica, induso dentro de la práctica se puede ver el viraje que toma el conocimiento en su formación hacia una conceptualización más acertada y organizada; para explicarlo de una mejor manera, esta idea la expresaremos de la siguiente forma, la forma de actuar o la actividad que ejercemos va formando poco a poco como pensamos, cuando conocemos lo hacemos de dos maneras, la primera de forma sensorial, es decir, no conocemos o entendemos la leyes que rigen esta actividad o su esencia, en esta forma el hombre apenas conoce los diversos aspectos del conocimiento pero aún no puede formar conceptos, que corresponde a un nivel más profundo, ni sacar condusiones lógicas. En la otra forma de conocimiento surge a través de un salto que realiza el ser humano, es decir, en la forma racional, el hombre comprende la esencia o las leyes que rigen su actividad, formando conceptos y sacando condusiones lógicas, entre los sensorial y lo racional, existe diferencias aualitativas como cuantitativas, y en este sentido las diferencias promueven el salto a que los aspectos de la realidad se relacionen y comprendan, en resumidas palabras se comprende las contradicciones intemas de las cosas objetivas (Mao, 1937).

Pero el conocimiento no se estanca en este sito, sino que tiene que demostrarse que tales condusiones lógicas son reales, y solo se comprueban en la práctica, pero es una práctica transformadora, es decir, auando cambiamos las cosas, a partir del conocimiento racional, podemos entonces inferir que el conocimiento generado es correcto y consecuente; es tan importante la práctica que sustenta todo el proceso del conocimiento del hombre, siendo su fuente y su consecuencia; si algún tipo de conocimiento no sale de la observación objetiva de la realidad, si no tiene una causa material, este conocimiento no es valorado, si algún tipo de conocimiento no se comprueba cambiando la misma realidad objetiva, entonces no sabremos si lo que generamos es correcto o no. 
Con esto no afirmamos la idea errónea de explicar o describir solo lo que vemos, ya que si no lo vemos o sentimos no es real, la teoría son ideas abstractas y como tal sus hipótesis son abstractas de igual forma, y sus explicaciones deben trascender a lo que simplemente sentimos o vemos, sin embargo, esta abstracción debe tener su aplicación práctica, sino, no debemos confiar que lo construido en la teoría es real y consecuente; en este sentido el conodimiento no está consumado, el hecho de que una teoría se acerque en explicar la realidad, no significa que no pueda surgir otra teoría que explique de una mejor manera las cosas. Como ejemplo de nuestra idea está la teoría cuántica, que más abstracto que pensar que una equación netamente matemática e imaginaria pueda explicar de forma tan aproximadamente acertada el comportamiento de los átomos y moléculas, cómo con la teoría cuántica pudimos entonces poder medir el diámetro y el radió atómico, cuando en esa época no teníamos los instrumentos para realizarlo, y gracias a esta teoría logramos crear una tecnología que jamás hubiéramos podido imaginar.

¿Qué tipo de prácticas existen actualmente en la sociedad? En esta fase de la humanidad y gracias a su desarrollo podemos esdarecer qué tipo de prácticas se presentan en la humanidad, aunque ya las señalábamos anteriormente no indicábamos cual es la práctica de importancia en el tema educativo; estas prácticas las podemos resumir en tres que son, la producción, las relaciones sociales con sus matices y la experimentación científica; dentro la experimentación cient́fica encontramos el oficio de los intelectuales y más espećficamente de los estudiantes, pero estos tres tipos de prácticas están íntimamente relacionada e influenciadas, sin embrago, es la producción la que determina a las otras dos y por ende la experimentación cient́fica aunque se puede prácticar en cualquier ámbito de la sociedad, son los intelectuales los que se hallan ligados a ésta, y su producción intelectual inevitablemente va a repercutir en la forma de producción y su distribución, al igual que repercute en las relaciones sociales con sus matices (Mao, 1937).

En la unidad didáctica presentada se busca entonces que los estudiantes miren, superficialmente las relaciones sociales existentes en su sociedad, ligadas con su actividad económica, partiendo de esto el estudiante debe idearse la forma en que su conocimiento teórico-práctico sea comprendido y si es posible aprovechable para la comunidad, por ello es necesario que se trabaje y en su elaboración utilizando todas las herramientas desarrolladas en su proceso de aprendizaje.

¿La RP que problemas tiene $y$ cuál debería ser su solución posible? Estas dificultades que presenta la RP, se pueden enumerar de la siguiente manera; a) falta de suficientes conocimientos teóricos en lo conceptual y procedimental, generando desinterés en los estudiantes, esto se expresa en que los estudiantes solo ven que estos problemas se van solucionar detrás de un algoritmo utilizado y que no es necesario entonces comprender la esencia misma del fenómeno, existen caos en que los estudiantes después de haber solucionado un problema en la parte teórica no comprende nada cuando van a aplicarlo, ya que se queda en un mecanicismo que no implica una comprobación real de sus teorías; b) la solución de problemas, se estanca en la extensión o "adecuación" de una solución estandarizada a una situación concreta, permitiendo entonces que el estudiante no dé el salto hacia su objetivo (Mora, García, 2005). Aunque la unidad didáctica presentada no genera grandes cambios en este sentido, si orienta en la necesidad de profundizar un poco la problemática social que genere la necesidad de adoptar una actitud hacia la utilización del conocimiento de una forma innovadora y aprovechable. c) los problemas son presentados de una forma tal que los estudiantes asumen que el problema debe tener una solución inequívoca, y por lo tanto el quid del asunto es encontrarla y no construirla, presentando de alguna forma como una ciencia que jamás se equivoca y que la construcción científica del conocimiento es un procesos lleno de éxitos; d) finalmente esta el escaso pensamiento hipotético-deductivo de los estudiantes (Mora, Garcáa, 2005).

¿Qué modelo de RP se debería aplicar en nuestra situación concreta? Bajo los anteriores argumentos, es apenas lógico que propongamos entonces un modelo, basado en la RP, para entonces generar realmente un salto cuantitativo y cualitativo en el aprendizaje y la enseñanza de 
la química en particular y de las ciencias en general, para ello nos basamos en las siguientes posiciones:

$>$ La enseñanza y el aprendizaje de la química debe estar ligada a la actividad productiva de las comunidades, es decir, la enseñanza y el aprendizaje de la química debe estar orientada según las necesidades de producción, donde intervenga la química, que tiene cierta población determinada; para ello es necesario conocer la situación particular de la comunidad, empezando desde ámbitos políticos, económico y culturales.

> La enseñanza y el aprendizaje de la química, debe estar orientada a hallar soluciones, a resolver problemas, esto implica fundamentación teórica fuerte y una práctica aun más fuerte, práctica enmarcada dentro de la experimentación científica, con un objetivo de hallar la solución de un problema real, no uno imaginario; con esto no solo nos enfocamos en el acceso prematuro a una resolución de un problema de las características descritas, sino más bien nos referimos a un proceso que apunte hacia este objetivo.

> La enseñanza y el aprendizaje de la química, debe ser provechoso para la comunidad, y en este sentido debe ser revestido de un carácter que beneficie a la mayoría de la comunidad, para esto es induso necesario tener claro qué tipo de relaciones existe en la vida cotidiana de la comunidad y si corresponde a una relación justa o injusta; orientando y complementado con un llamado hacia la solución de problemas comunitarios que no coloque o justifique la dominación de la economía sobre la vida diaria de la comunidad.

$>$ Las aplicaciones prácticas deben ser constantemente revaluadas, mientras las condiciones lo exijan y permitan, de esta manera la imagen de la ciencia no debe quedarse en una imagen exitosa y estática, sino en un proceso de mejoramiento en movimiento constante.

¿Qué esperamos con la unidad didáctica? La forma en que se plantea la unidad tiene aproximadamente la siguiente estructura: 1) una introducción que haga saber al estudiante que el conocimiento de los gases no solamente es un concepto teórico sino de aplicación concreta, teniendo su importancia en el rol que juega en la sociedad, para ello se introduce algunos datos que hagan pensar al estudiante que está ubicado en un contexto y que de alguna manera el concepto de gas, puede estar relacionado e induso aporte algunas soluciones a los problemas existentes. 2) historia y marco teórico; en esta parte exponemos paralelamente la historia del concepto como la explicación teórica de los gases y su aplicación general, mostrando ecuaciones y su posible aplicación vista desde su construcción histórica, para hacerlo ameno se planteó en forma de auento. 3) elaboración de un programa de actividades, que plantee un desarrollo desde las sensaciones hasta un conocimiento racional un poco aproximado al científico, incluyendo una actividad práctica con la comunidad, enmarcado en una feria cient́fica, que apunte a generar una base de datos accesible para la comunidad y sea provechosa para la solución de problemas concretos, además, induye la necesidad de que el estudiante haga el ejercicio de explicarle de una forma comprensible un experimento que el mismo realizo y montó.

\section{Conclusiones}

$>$ En el proceso de conocimiento lo principal es la práctica, es el sustento material y de igual forma es la consecuencia inmediata del conocimiento, es por ello que la RP, es un reflejo de esto porque lleva a practicar al estudiante bajo sus condiciones materiales; lo que le falta entonces es revestir esta práctica como una práctica transformadora, y no contemplativa como se maneja actualmente.

$>$ En el proceso de enseñanza-aprendizaje, se enmarca dentro de la práctica social, conocida como experimentación científica, la transformación de esta experimentación científica debe conllevar a un cambio en el proceso educativo y por ende un cambio cultural que formen personas integras.

$>$ El proceso de enseñanza-aprendizaje debe estar orientado según las necesidades de la comunidad, apuntando a su solución y apropiación de la comunidad de este conocimiento generado. 
$>$ EI proceso enseñanza-aprendizaje, debe ser revaluado según cambie las condiciones contextuales de la comunidad.

\section{Bibliografía}

Marx, C. Engels, F. (1846) Feuerbach: Oposición entre las concepciones materialista e idealistas; Capitulo I de la Ideología Alemana. Alemania. Edición Lenguas Extranjeras Pekín.

Mora, W. Garcáa, A. (2005). La resolución de problemas: una línea de investigación en las enseñanzas de las ciencias. Revista educativa Voluntad, 15-19.

National Academy Press. (2000). CAPITULO 2: LA INDAGACIÓN EN LOS ESTANDARES DE LA CIENCIA. unw.eduteka.org.

Tse Tung, M. (1963). ¿De dónde provienen las ideas correctas?. Peḱ́n, China. Ediciones en lenguas extranjeras Pekín.

Tse Tung, M. (1937). Sobre la práctica. Pekín, China. Ediciones en Lenguas Extranjeras Pekín. 